\title{
Climate Change, Local Weather and Customized Early Warning Systems based on Biometeorological Indexes
}

\author{
Pablo Fdez-Arroyabe ${ }^{1,2}$ \\ 1. Department of Geography, University of Cantabria, Avda. Los Castros S/N 39005, Santander, Spain \\ 2. President-Elect of the International Society of Biometeorology
}

Received: February 15, 2015 / Accepted: March 05, 2015 / Published: March 20, 2015.

\begin{abstract}
The following paper is a brief theoretical approach to the state of the art in relation to the new demands of society in the area of climate and weather-related services for health. Apart from the study of the impacts of climate change on health which requires a longer consideration, a special emphasis is given to the area of weather-related services based on biometeorological forecasting. A new way of understanding the interaction between atmospheric factors and living organisms is presented and some biometeorological indexes already developed are shown. Customized biometeorological services must be constructed in the field of human health based on the ICTs development and the amount of climatic data available but new approaches and scientific methods are required to success in achieving this enterprise.
\end{abstract}

Key words: Climate service, variability, biometeorology, customized warning systems.

\section{Introduction}

The main goal of this article is to show a new approach to the understanding of climate and weather related human health risks. Meteorological factors have acted as determinants of human health crisis for ages. Climate change and its associated extreme events are confirming an ancestral relationship between nature and living organisms based on ecological and homeostatic rules. They have been taken into account very poorly by contemporary societies in order to mitigate climate-related impacts.

In this sense, the development and implementation of new climate services from international and local organisms should be a must such as the GFCS (Global Framework for Climate Services), the WMO (World Meteorological Organization) priorities [1] are shown in Fig. 1:

(1) Agriculture and food security have become a

Corresponding author: Pablo Fdez-Arroyabe, professor, research fields: biometeorology, geographic information technologies, physical geography and environmental sciences. E-mail: fernandhp@unican.es. major concern. Vulnerability of this sector is extremely dependent on atmospheric variability and change. (2) Natural hazards are mainly caused by weather and climate. Building a greater resilience against hydro-meteorological risks is essential. (3) Drinkable water access is fundamental for human development. Meteorological uncertainties about its availability should be minimized and accessibility to it should be improved in those regions of the world where its scarcity is normal. (4) Public health depends on climate variability and change. The outbreak of new emerging infectious diseases is connected to demographic and environmental changes. Transmission vectors can modify their spatial distributions if new environmental conditions are generated. Individual health can also be associated to meteorological changes and anomalous weather variability.

\section{Atmospheric-Related Health Risks}

\subsection{Weather Variability and Climate Change}

Weather relates to the state of the atmosphere on a 


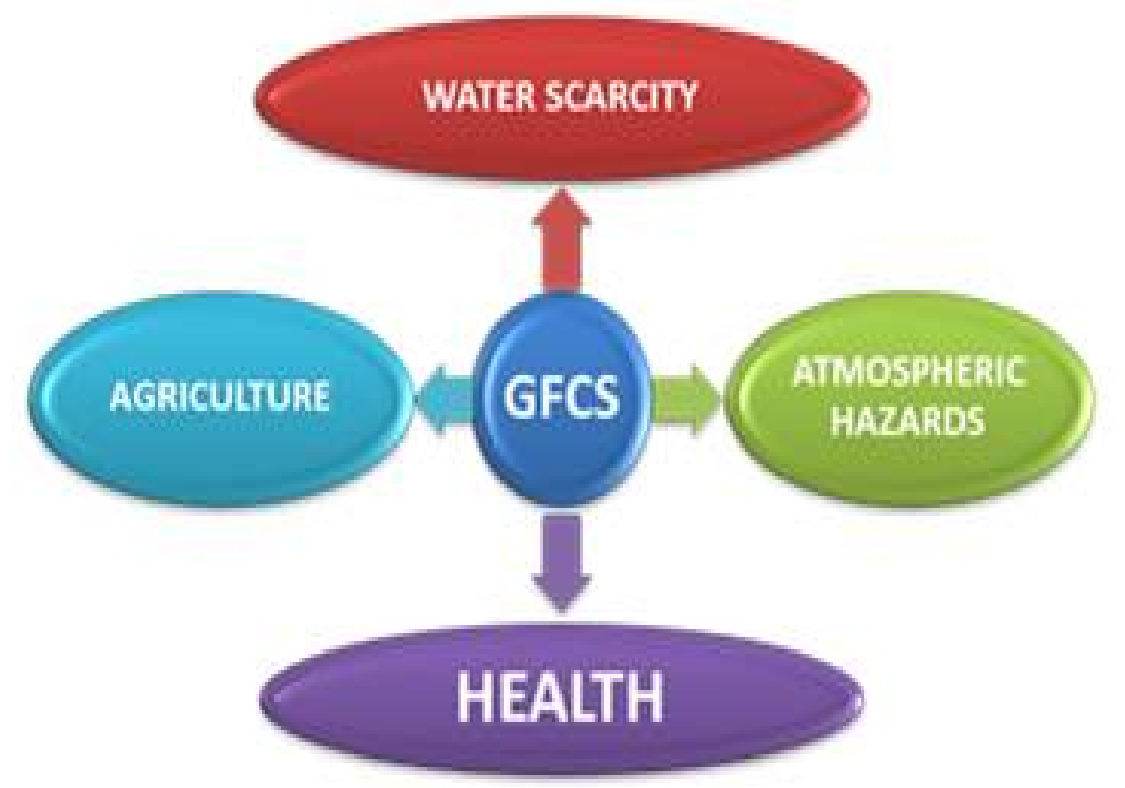

Fig. 1 Priority areas for the development of climate services. Source: World Meteorological Organization.

particular day or sequences of few days in a place on the earth. Climate is related to the average state of the atmosphere for long periods of time (30-40 years) in a particular place. In this sense, the concepts of weather variability and climate change become essentials to speak about the atmospheric determinants of human health. The first assumption is that weather variability itself is strong enough to impact on human beings health without even considering climate change.

Atmospheric extreme events are part of the atmospheric patterns. According to the different reports of the IPCC (Intergovernmental Panel for Climate Change), climate change will increase the number of extreme events and their persistence and intensity. The study of atmospheric-related health risks admits two different approaches:

- Attending specifically to climate change impacts. In this case, health risks are related to the increase in number, duration and intensity of extreme events in some regions of the planet and also to the generation of new emerging diseases in new environments:

- Attending to anomalous weather variability and change that are not extreme events. There are atmospheric situations that are not extreme and have a severe impact on human's health.

Due to the fact that these atmospheric contexts are more frequent than extreme atmospheric events, their impact on human health should also be assessed. Climate and weather-related health risks (Fig. 2) depends on the vulnerability of each person: (1) to the new environmental scenarios and extreme events as a consequence of climate change and (2) to the normal anomalous weather impacts on human health.

\subsection{Biometeorological Profiles and Health Risk}

Biometeorology has studied the interaction between the atmosphere and living organism for more than 60 years. Plants, animals and human Biometeorology are the three main subdivisions of this scientific discipline that works with a specific concept of health based on an ecological approach. Each meteorological variable is constantly having an impact on living organisms.

Air humidity, atmospheric pressure, sun radiation, ozone levels, electromagnetic fields and many more physical and chemical variables are constantly interacting on the ecosystems.

From a biometeorological point of view, each person is constantly assuming the risk of having a 


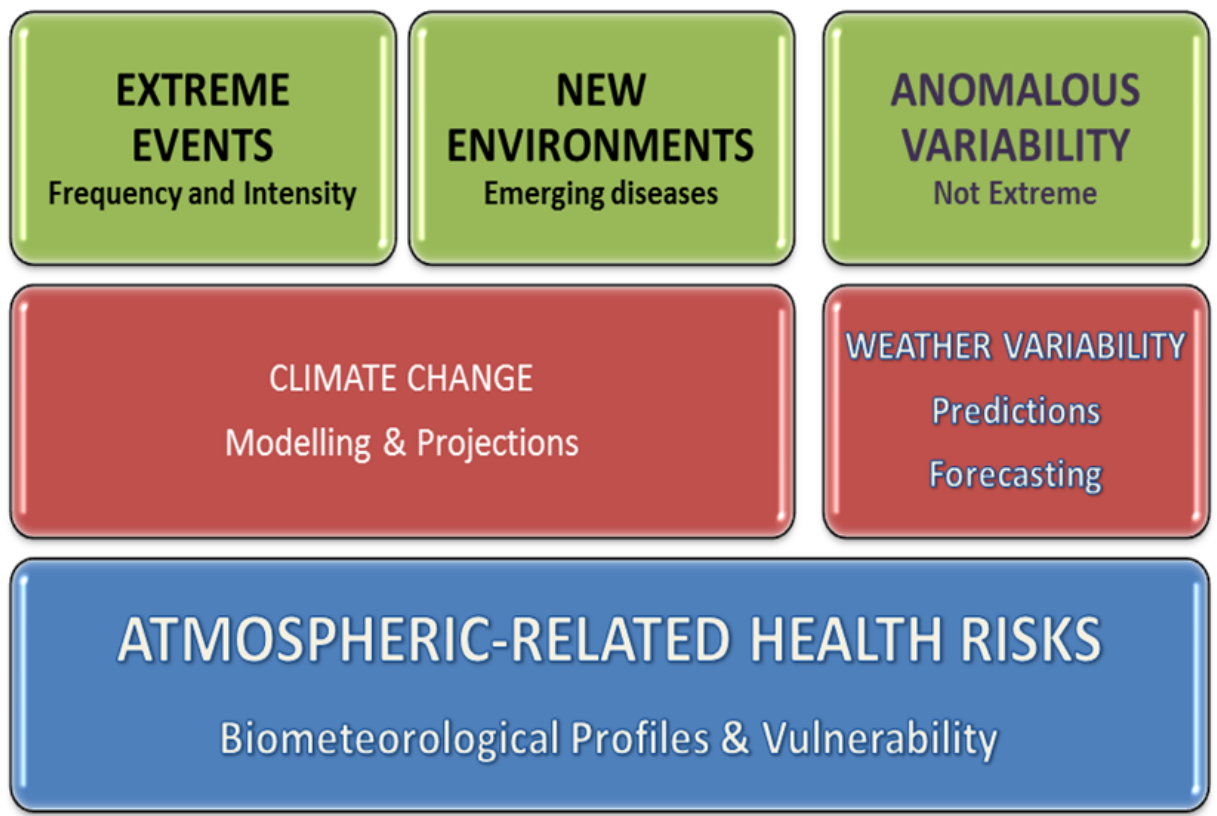

Fig. 2 Climate and weather related health risks.

health crisis because any living organism is permanently reacting to atmospheric variability and change. These interactions allow people to develop their own biometeorological profiles which are defined by the level of dis/confortability that each individual has been in relation to each atmospheric variable in the climatic domain where their inmune systems was developed.

Under this biometeorological definition of health, climate/weather-related risks explanation becomes a complex issue in which the vulnerability of individuals differs according to each biometeorological profile. In this sense, processes that take place at different temporal scales such as physiological thermoregulation, acclimatization and biological and cultural adaptation defined by Burton et al. [2] are related to minimizing the impacts of atmospheric changes and variability on health.

\section{Early Warning Systems}

Biometeorological early warning systems are not just meteorological forecasting systems. Meteorological offices are able to forecast extreme events but, at the same time, many other statements and public departments are required in order to offer an alarm and to activate protocols of social assistance and to inform to the public opinion. This is something that has been implemented in relation to natural hazards in many countries but it is something to do in the future in relation to the atmospheric determinants of human health risks. Some examples of EWS based on biometeorological indicators are briefly shown afterward and different results for each system too. They can be applied to different scales and to different groups of diseases.

\subsection{Global Early Warning Systems based on Atmospheric Oxygen Variation}

The development of biometeorological warning systems at global level is not something new. Some indexes such as the DOA Index, developed by Lecha et al. [3, 4] are offering daily forecasting to different regions of the world. The index expresses the variation of the amount of oxygen in the atmosphere between consecutive days and it has been proved to be linked in different ways to excess morbidity in Northern Spain and Cuba for different groups of diseases (brain-vascular, cardio-vascular and 
respiratory diseases) according to the ICD (International Classification of Diseases).

\subsection{MCI (Meteorological Contrast Index) and Influenza Epidemics}

A different research based on the MCI estimation, developed by Fdez-Arroyabe [5] has been applied to the study of influenza in the Iberian Peninsula and on the East Coast of USA. Previous studies show a synchronicity between the highest flu rates increments and MCI values. The existence of a delay period between infections and the diagnose offers researchers the chance to incorporate a biometeorological approach to the traditional epidemiological and statistical ones in the study of influenza. The definition of a biometeorological warning system will allow us to point out levels of risk of experiencing an outbreak or a spreading of this infectious disease. The obtained results for the Iberian Peninsula [6] for the most important epidemic periods between 2000 and 2010 have indicated that climatic domains and meteorological contrast are relevant factors in the process of the influenza epidemics expansion.

In this sense a similar approach has been recently taken on the East Coast of USA where different epidemic periods has been analyzed by considering the SSC (Spatial Synoptic Classification) [7] of weather types defined by Sheridan et al. [8] as input for the index.

Preliminary results have confirmed that the meteorological contrast is a physical factor which can indirectly trigger the outbreak of the epidemics and can promote the expansion of the diseases. None epidemiological model is taking into account nowadays this approach in order to manage influenza spreading.

\subsection{Customized Biometeorological Early Warning Systems based on Technological Development}

The definition and implementation of weather-related CEWS (Customized Early Warning Systems) at an individual level is linked to the biometeorological profile of each person and has been developed based on the ecological explanation of human diseases mentioned before. The biometeorological profile of individuals can be defined attending those environmental conditions that affected each person while his immune system was developed.

This definition can be highly complex. Nevertheless, a multivariate approach can take us to define specific comfort thresholds, biometeorological impacts are low, medium or high for each person in relation to each variable. Customized Early Warning Systems can easily be spread all over the world through the development of new climate services based on ICTs technologies [9] such as WEB Services, GIS or APPs.

\section{Results and Discussion}

\subsection{DOA Index Global Forecasting}

An example of the DOA biometeorological index is presented for Central and North America (Fig. 3) where hypoxia and hyperoxia levels are represented cartographically. Maps show the positive and negative variation of the content of oxygen $\left(\mathrm{gr} / \mathrm{m}^{3}\right)$ in the atmosphere for a period of 24 hours. These maps are automatically generated by the GEOBIOMET Research Group at the University of Cantabria, Spain every six hours for the whole world.

Blue colors are indicating an important change towards hyperoxia conditions while green colors would suggest a change promoting hypoxia conditions. Due to the differences in latitude and longitude, hyperoxia and hypoxia environments must be understood in relative terms. Magnitude of changes in the index should be evaluated considering normal variability of each climatic zone in order to identify real anomalies. A second cartographic representation of the DOA index is presented below (Fig. 4) for the regions of Africa and Middle East in order to show the differences in color intensity in relation to the previous map. Neutral level indicates there is no a relevant change in the content of oxygen in the last 24 hours. 
DOA INDEX ON CENTRAL AND NORTH AMERICA

From January $22^{\text {th }}$ to $23^{\text {th }}$ (Year 2015)

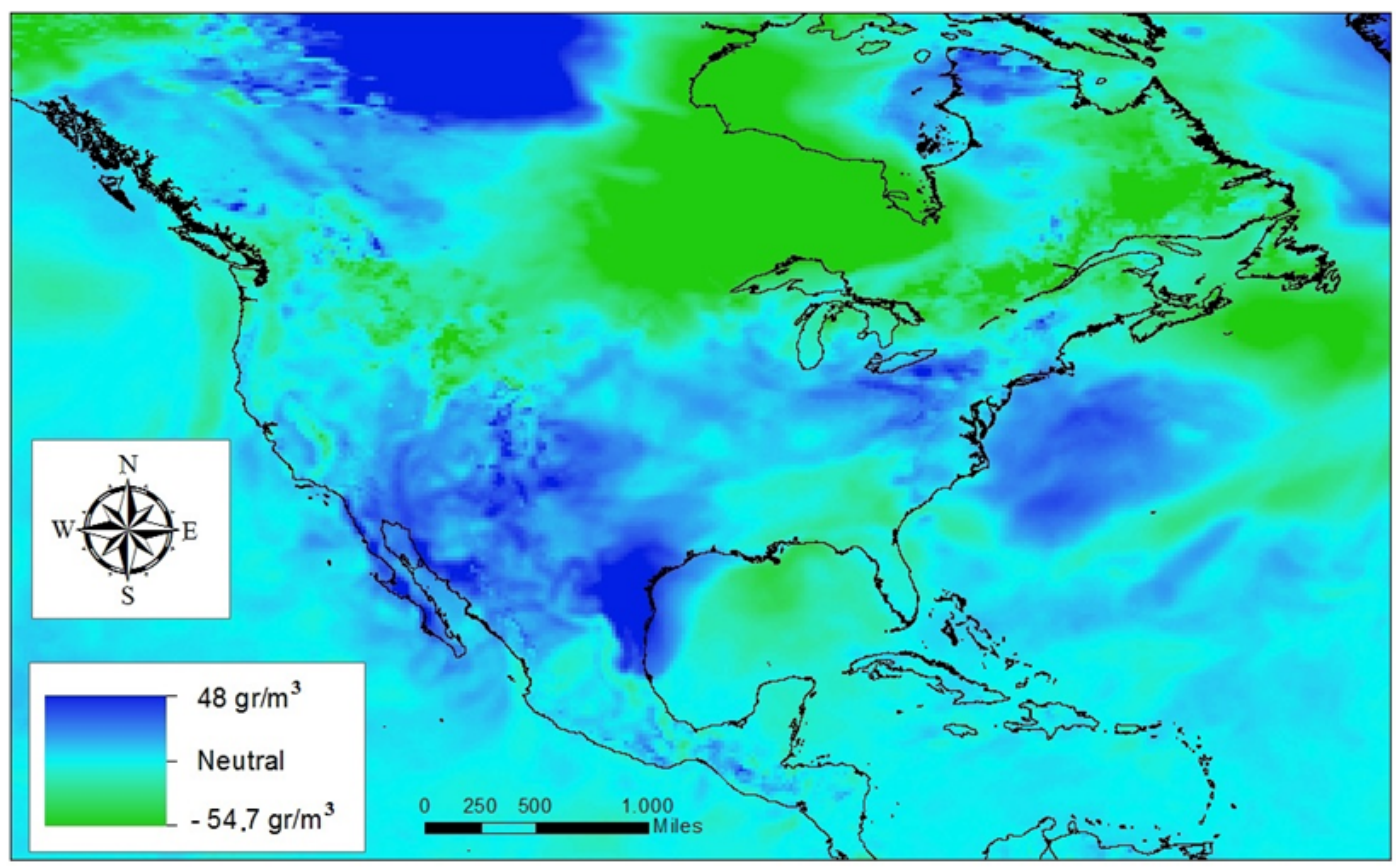

Fig. 3 Variation of DOA Index for North America and Caribe calculated from NOAA-GFS datasets.

DOA INDEX CONTRAST ON AFRICA AND MIDDLE EAST From January $22^{\text {th }}$ to $23^{\text {th }}$ (Year 2015)

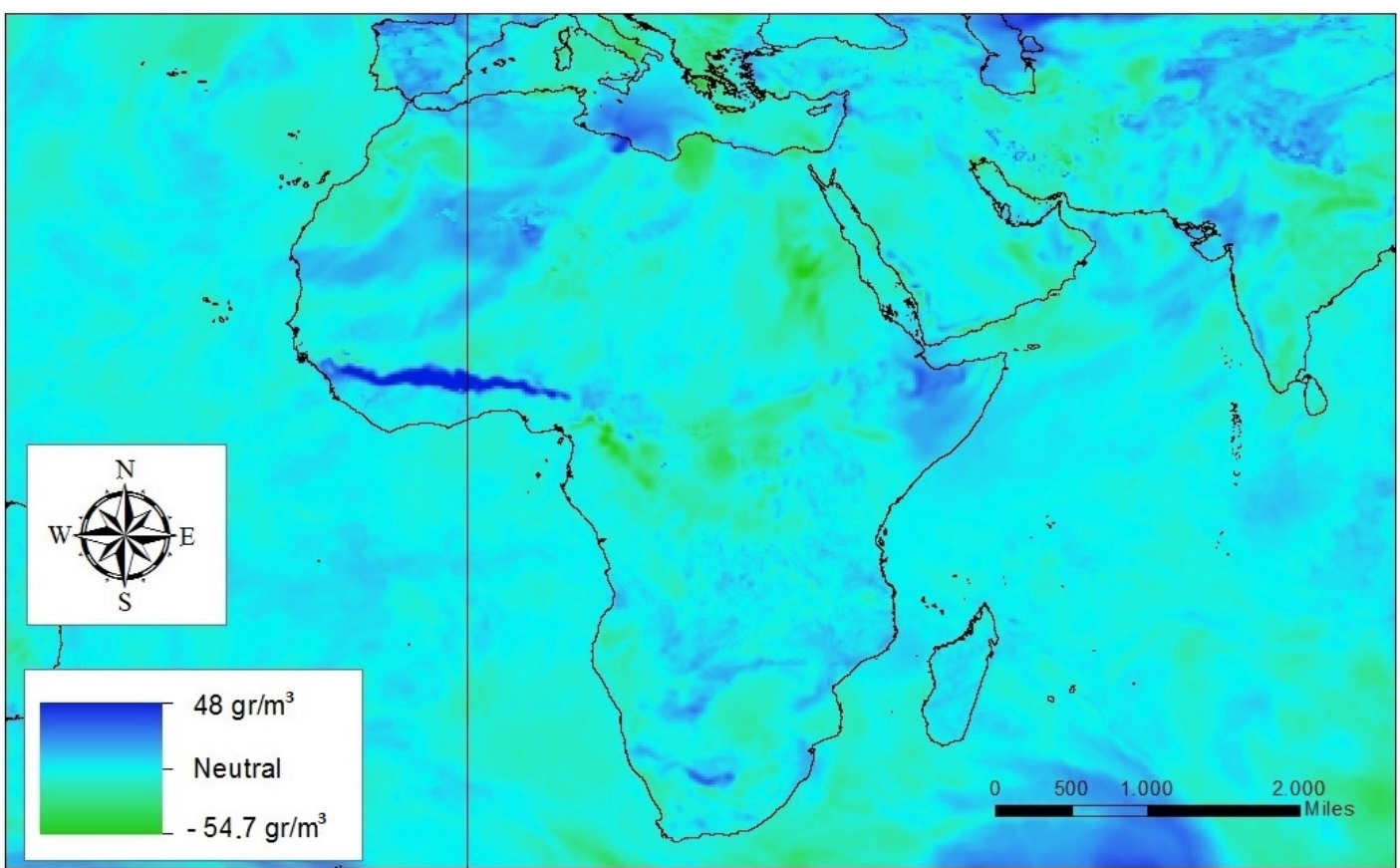

Fig. 4 Variation of DOA Index for the regions of Africa and Middle East calculated from NOAA-GFS datasets. 
In consequence, there is no biometeorological distress on human health due to oxygen changes. Extreme hyperoxia conditions have been associated in Northern Spain and Cuba to some respiratory diseases such as Asthma while extreme hypoxia levels have a more fuzzy impact associated to cerebral vascular diseases $[10,11]$. Apart from these, extreme changes have an impact on morbidity at the emergency rooms in the hospitals and can also be linked to anomalous mortality.

\subsection{Influenza Forecasting based on ICM}

A second example of one biometeorological index based on the idea of atmospheric contrast is the MCI. This index calculates three parameters using as input different weather types classifications. In this particular case, the index parameters were related to the influenza rate evolution in the Iberian Peninsula for one of the most important epidemics between year 2004 and 2005 during the 21thcentury.It can be seen how parameter P1 and P2 reached the highest value one week before the initial spreading of the epidemic. The third parameter called MCI takes the highest value on the week where the biggest increment took place. Later on, values of flu rate keep increasing due to the normal epidemiological trend of the disease. This pattern has also been confirmed for the Sanitary Area of Philadelphia where biometeorological distress produced by atmospheric contrast was considered a relevant factor in relation to the spreading of the disease during the study periods (2001-2007). In this study, MCI weekly meteorological values vary from 35 in the region of Boston to 24 in the area of Atlanta. Once again, biometeorological impacts must be considered a relative concept that makes sense in each specific climatic domain where influenza epidemic takes place. The spatial variability of meteorological variables becomes essential to define levels of risk. Table 1 is presenting the values of the three parameters that are computed by the MCI. First parameter (P1) represents how diverse weather types are on a specific period of time. Second parameter (P2) indicates the number of outbreaks that are found on the sequences of daily weather types that are defined for each epidemiological week, and finally the third parameter, MCI, quantifies the magnitude of the changes in the epidemiological week.

There is no a direct relationship between a high level of contrast and the outbreak of an epidemic but an elevated contrast can help the spreading process. Many epidemiological and microbiological issues must also be considered in order to complete a rational explanation of the role of the atmospheric contrast on starting and promoting influenza epidemics.

\subsection{Customized Biometeorological Early Warning Systems based on Technological Development}

Finally, an example of a Biometeorological Early Warning System based on a mobile application is presented. The BETA version of the App OxyAlert

Table 1 MC Index and influenza rate increments per epidemic week in the outbreak of 2004-05 in the Iberian Peninsula. Source: Spanish Epidemiological National Center

\begin{tabular}{llllll}
\hline Epidemic Week & $\begin{array}{l}\text { Rate } \\
(\%)\end{array}$ & P1 & P2 & MCI & $\begin{array}{l}\text { Rate } \\
(\boldsymbol{\Delta})\end{array}$ \\
\hline 47 & 22.08 & 2 & 1 & 90 & -7.43 \\
48 & 30.91 & 4 & 3 & 270 & 8.83 \\
49 & 27.61 & 4 & 3 & 248 & -3.29 \\
50 & 67.64 & 4 & 3 & 226 & 40.02 \\
51 & 87.88 & $\mathbf{4}$ & $\mathbf{4}$ & 382 & 20.24 \\
52 & 195.13 & 3 & $\mathbf{4}$ & $\mathbf{4 9 5}$ & $\mathbf{1 0 7 . 2 5}$ \\
1 & 355.72 & 2 & 1 & 90 & 160.58 \\
2 & 542.86 & 2 & 1 & 90 & 187.14 \\
\hline
\end{tabular}




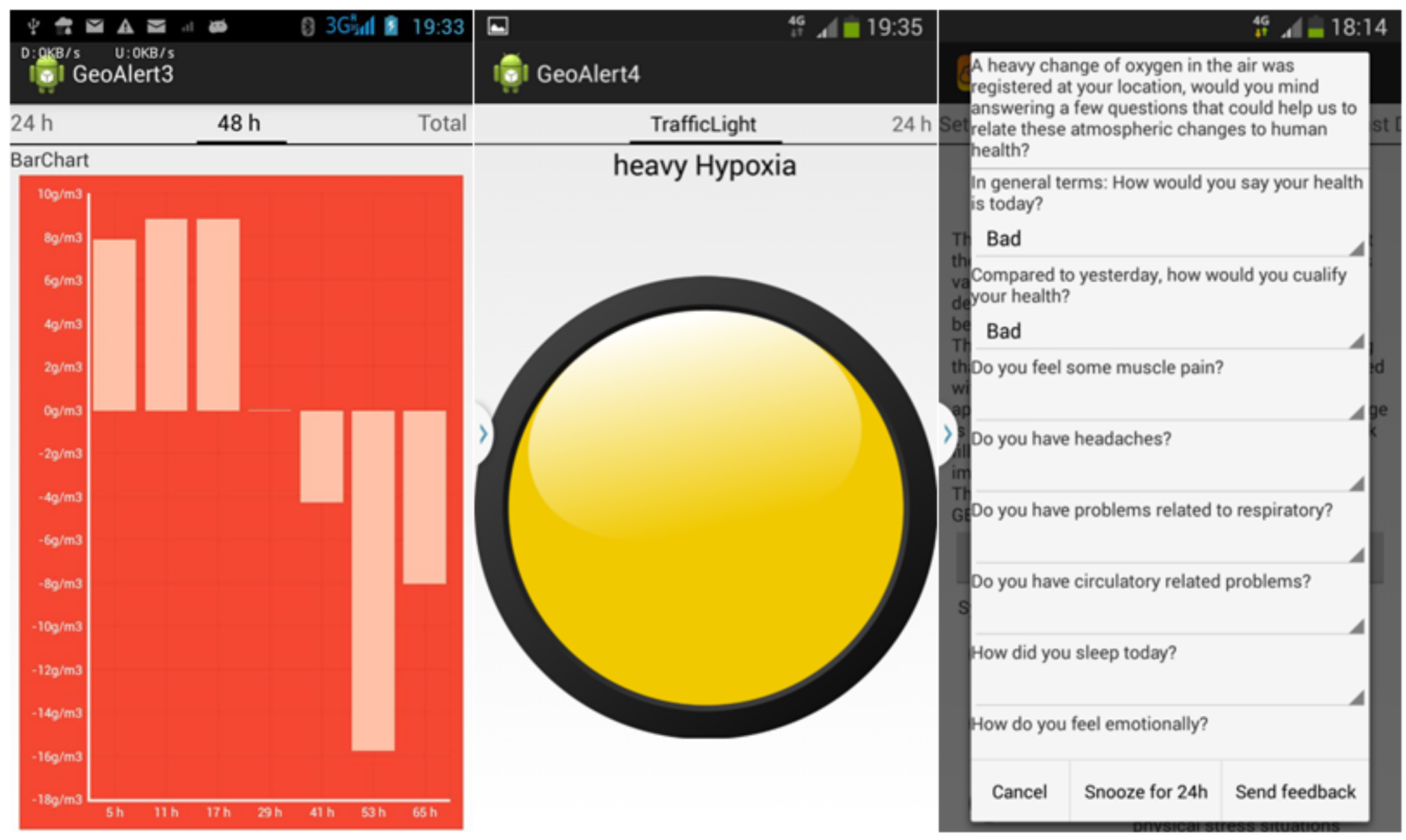

Fig. 5 Screenshots of the mobile App (application) OXYALERT developed by the GEOBIOMET Research Group at the University of Cantabria, Spain 
has been recently published by GEOBIOMET Research Group at the University of Cantabria, Spain. This new App is linked to the hyperoxia and hypoxia levels of the DOA index presented before and works under the Android operating system. The application is now being assessed by a group of testers and OxyAlert BETA is freely available [12] and offers alerts at different levels of hypoxia and hyperoxia conditions all over the world. There are risk levels (Extreme, Very Heavy, Heavy, Moderate, Slight and Neutral) based on previous studies that are associated to different impacts on groups of diseases.

The app forecasts the impact 72 hours in advance for the place where the user is located (Fig. 5a). The service is using the geo-localization properties of the mobiles phones in order to offer this service all around the Earth and is also offering an alert to the user when levels of Extreme, Very Heavy or Heavy are reached (Fig. 5b). At this time, the users are asked to fill in a questionnaire with eight questions related to their physical and emotional health state (Fig. 5c). This information is collected automatically on a biometeorological relational database management system in order to assess and improve the biometeorological model in the future.

\section{Conclusions}

The amount of information related to Meteorology and Climatology has never been so important before. The scientific community has created networks among researchers from all over the world in order to study climate change and its future impacts (IPCC) on multiple fields. The social dimension of climate change as a global problem is constructing a constantly growing awareness about environmental problems on the Earth.

There is no controversy any more about the idea that people's wellbeing is dependent on ecological services and respect to nature. This fact becomes even more relevant when human health is mentioned.
Climate and weather-related services based on ICTs (information and communication technologies) can be developed for many groups of potential customers in the health sector. Nevertheless, access to meteorological datasets is not enough. The development of new services will be only possible if new methodological approaches about how nature and living organisms are connected are developed. Information should be raw material to create new knowledge and technology the tool to improve people's life.

\section{Acknowledgements}

Funding support has been received from the Research Directorate of the University of Cantabria and from the Spanish Minister of Economy and Competiveness through the National Funding Budget applied to the projects CSO2013-46153-R. Both institutions are supporting the GEOBIOMET Research Group activities on the field of Biometeorology.

\section{References}

[1] World Meteorological Organization. 2014. Implementation Plan of the Global Framework for Climate Services. Access June 14, 2014. http://www.wmo.int/gfcs/.

[2] Burton, I., McGregor, G., and Ebi, K. L. 2009. Biometeorology for Adaptation to Climate Variability and Change. Berlin, Germany: Springer Netherlands.

[3] Lecha, L. 1999. "Effects of Climate Variability on the Health of the Cuban Population." Bulletin of the World Meteorological Organization 48 (1): 18-22.

[4] Lecha, L., Fdez-Arroyabe, P., and Martín, D. 2011. "The Global Monitoring of Meteor-Tropic Effects on Human Health Such As Fundamental to Mitigate the Potential Impacts of Climate Change on Health and Society." In Proceedings of the 7th European Congress on Tropical Medice \& International Health, 43-4.

[5] Fdez-Arroyabe, P. 2004. "Climate, Weather and Flu Diagnoses Incidence in the Region of Santander during the 1999-2000 Epidemic Diffusion Period.” Aerobiologia 20: 223-8.

[6] Fdez-Arroyabe, P. 2012. "Influenza Epidemics and Spanish Climatic Domains." HEALTH4: 941-5.

[7] Sheridan, S. C., and Kalkstein, L. S. 2004. "Progress in Heat Watch-Warning System Technology.” Bulletin of 

based on Biometeorological Indexes

the American Meteorological Society 85: 1931-41.

[8] Sheridan, S. C. 2002. The Redevelopment of a Weather-Type Classification Scheme for North America. Int. J. Climatol. 22: 51-68.

[9] Fernández de Arróyabe, P. 2013. "Climate Services and Human Health: a Niche of Opportunities for Economic Growth." Scientific Annals of Alexandru IoanCuza. Geography Series 59 (2): 135-52.

[10] Fdez-Arroyabe, P., and Lecha, L. 2008. "Assessment of Two Biometeorological Early Warning Sytems Basedon
Contrast in Northern Spain." In Proceedings of the International Conference of the Spanish Society of Climatology, 781-92.

[11] Fdez-Arroyabe, P. 2013. "Meteorological Conditions and Human Health." In Adverse weather in Spain, edited by Legaz Martinez, C, and Valero Rodriguez, F. Madrid, AMV Editions.

[12] Fdez-Arroyabe, P. 2015. Mobile App OxyAlert BETA, https://play.google.com/store/apps/details?id=es.geobiom et.oxyalert. 